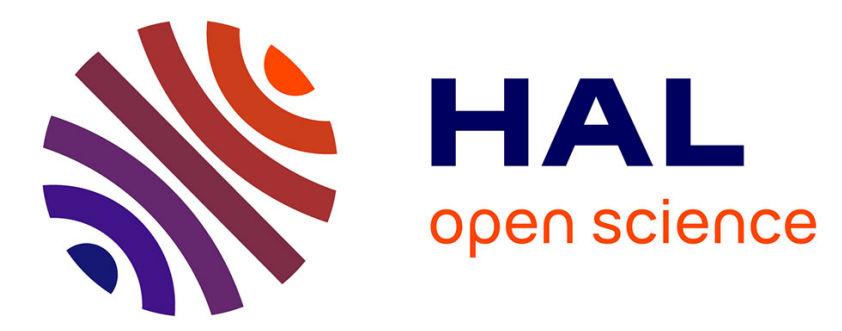

\title{
Structure of rat odorant-binding protein OBP1 at 1.6 angstrom resolution
}

\author{
Scott A. White, Loïc Briand, David J. Scott, Antoni J. Borysik
}

\section{To cite this version:}

Scott A. White, Loïc Briand, David J. Scott, Antoni J. Borysik. Structure of rat odorant-binding protein OBP1 at 1.6 angstrom resolution. Acta crystallographica Section D : Structural biology [1993..], 2009, 65 (5), pp.403-410. 10.1107/S090744490900420X . hal-01137039

\section{HAL Id: hal-01137039 \\ https://hal.science/hal-01137039}

Submitted on 30 Mar 2015

HAL is a multi-disciplinary open access archive for the deposit and dissemination of scientific research documents, whether they are published or not. The documents may come from teaching and research institutions in France or abroad, or from public or private research centers.
L'archive ouverte pluridisciplinaire HAL, est destinée au dépôt et à la diffusion de documents scientifiques de niveau recherche, publiés ou non, émanant des établissements d'enseignement et de recherche français ou étrangers, des laboratoires publics ou privés. 
Acta Crystallographica Section D

Biological

Crystallography

ISSN 0907-4449

\section{Scott A. White, ${ }^{a}$ Loïc Briand, ${ }^{b, c, d}$ David J. Scott ${ }^{\mathrm{e}}$ and Antoni J. Borysik $^{\mathbf{e}_{*}}$}

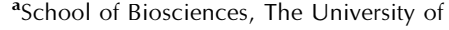
Birmingham, Edgbaston, Birmingham, England, bINRA, UMR1169 FLAVIC, F-21000 Dijon, France, ${ }^{\mathrm{C}}$ ENESAD, UMR1169 FLAVIC, F-21000 Dijon, France, ${ }^{d}$ Université de Bourgogne, UMR1169 FLAVIC, F-21000 Dijon, France, and ${ }^{\mathbf{e}}$ National Centre for Macromolecular Hydrodynamics, School of Biosciences, University of Nottingham, Sutton Bonington Campus, Loughborough, England
}

Correspondence e-mail: antoni.borysik@nottingham.ac.uk

\section{Structure of rat odorant-binding protein OBP1 at $1.6 \AA$ resolution}

The nasal mucosa is a specialist interfacial region sandwiched between the olfactory system and the gaseous chemical milieu. In mammals and insects, this region is rich in odorant-binding proteins that are thought to aid olfaction by assisting mass transfer of the many different organoleptic compounds that make up the olfactory landscape. However, in mammals at least, our grasp on the exact function of odorant-binding proteins is tentative and better insight into the role of these proteins is warranted, not least because of their apparent significance in the olfactory systems of insects. Here, the crystal structure of rat odorant-binding protein 1 is reported at $1.6 \AA$ resolution. This protein is one of the best-characterized mammalian odorant-binding proteins and only the third such protein structure to be solved at high resolution. The protein was crystallized in the holo form and contains an unidentifiable ligand that is probably an artefact from the Pichia pastoris expression system. Comparisons are made between this structure and a modelled OBP1 structure produced using the crystal structure of aphrodisin as a template. Comparisons are also made between OBP1 and the other two rat OBP subtypes, for which crystallographic data are unavailable. Interestingly, we also show that OBP1 is monomeric, which is in contrast to its previous assignment.

\section{Introduction}

Mammalian odorant-binding proteins (OBPs) are part of the lipocalin superfamily of proteins (Flower et al., 2000) together with proteins such as the mouse major urinary protein (Bocskei et al., 1992), bovine $\beta$-lactoglobulin (Kontopidis et al., 2002) and cellular retinoic acid-binding protein (Cowan et al., 1990). These proteins consist of an eight-stranded antiparallel $\beta$-barrel with a C-terminal $\alpha$-helix that is packed against its exterior. The buried side chains of the lipocalin $\beta$-barrel mostly originate from hydrophobic residues and form a central cavity or calyx that provides a favourable site for the transportation of apolar ligands in aqueous environments (Flower et al., 2000).

Mammalian OBPs were first isolated from the olfactory mucosa of cows and were characterized as proteins that have a specific affinity for 2-isobutyl-3-methoxypyrazine (bell pepper smell; Bignetti et al., 1985). Since then, these proteins have been found in the nasal mucosa of many other mammals, such as pigs (Dal Monte et al., 1991), rats (Briand et al., 2000), porcupines (Ganni et al., 1997) and humans (Briand et al., 2002). Certain animal species have been shown to express more than one form of OBP, with rats and porcupines being notable examples. Interestingly, each different form of rat
Received 29 October 2008 Accepted 4 February 2009

PDB Reference: OBP1, 3fiq, r3fiqsf.
C 2009 International Union of Crystallography Printed in Singapore - all rights reserved 
OBP has been shown to bind preferentially to ligands of a different chemical class (Lobel et al., 2002). This is important as it implies that OBPs could have more than just an ancillary role in olfaction and could be involved in the earliest stages of odour coding.

Although many different mammalian OBPs have been isolated, crystallographic data are only available for bovine OBP (OBPb; Bianchet et al., 1996; Tegoni et al., 1996) and porcine OBP (OBPp; Spinelli et al., 1998). OBPb was considered to be the archetypal OBP for several years and, as anticipated from biophysical analysis, the crystal structure of this protein showed that it was dimeric. Surprisingly, however, $\mathrm{OBPb}$ was an example of a domain-swapped dimer with the $\alpha$-domains of two monomers interchanging, a structural feature that is unique in the lipocalin protein family. In contrast to its bovine counterpart, OBPp is monomeric in solution. This is thought to be the consequence of the insertion of a single residue (Gly121) in the loop preceding the $\alpha$-domain in OBPp and also of the presence of a C-terminal disulfide bond which is absent in OBPb (Ramoni et al., 2002, 2008).

Despite the fact that OBPs are expressed at high concentrations (up to $1 \mathrm{mM}$ ), no biological function has yet definitively been ascribed to these proteins (Steinbrecht, 1998). A putative role as carriers of organoleptic compounds to the odour receptors (ORs) was initially proposed (Briand et al., 2000). In this model, it was conceived that OBPs functioned as a universal solvent, assisting olfaction by nonspecifically increasing the concentration of odorants in the nasal mucosa. However, although this carrier role is appropriate for OBPs, given the canonical transport role of lipocalins together with the physical locality of OBPs and the observation that they bind a range of volatile ligands in vitro in the low micromolar range (Briand et al., 2000, 2002; Lobel et al., 2002; Nespoulous et al., 2004), there is no direct evidence to support it. Furthermore, the activation of ORs has been observed in the absence of OBPs (Krautwurst et al., 1998). Thus, the specific role of OBPs remains to be properly ascertained, although several different models have been put forward. Matarazzo and coworkers showed that porcine OBP (OBPp) interacts strongly $\left(K_{\mathrm{d}}=9.5 \mathrm{n} M\right)$ with human OR17-210 in vitro. From this, they postulated that OBPs have a dual role, with their specific function being driven by the concentration of odours in the mucous layer (Matarazzo et al., 2002). Matarazzo and coworkers suggested that at high ligand concentrations OBPs act as scavengers averting OR saturation, whereas at low concentrations they act as couriers, actively delivering odorants to the ORs. More recently, Vidic and coworkers also demonstrated a specific OBP-OR interaction in vitro and put forward a more detailed role for OBPs than that postulated by Matarazzo and coworkers (Vidic et al., 2008). Vidic and coworkers demonstrated that the response of OR17-40 to helional diminished significantly at high odorant concentrations in the absence of OBP1. Vidic and coworkers postulated that ORs function as homodimers, with each monomer in the pair possessing specific binding sites for odorants and OBP. OR dimers are active only when bound to a single odorant. Since each OR monomer cannot accommodate both odorant and OBP simultaneously, Vidic and coworkers suggest that OBPs act to preserve the active state of the OR dimer at high odorant concentrations. Contrary to this idea, Taylor and coworkers recently proposed that OBPs function to slow down the kinetics of odorant release from the nasal mucosa (Taylor et al., 2008). Prompted by the observation that ORs can integrate odorant stimulus (Firestein et al., 1990), this idea has OBPs functioning as odorant reservoirs, permitting the detection of transitory stimuli which may otherwise be undetectable in their absence.

OBP1 is an $18 \mathrm{kDa}$ protein that is one of the best characterized mammalian OBPs and that has been the subject of many ligand-binding and unbinding studies both in vitro and in silico (Lobel et al., 2002; Nespoulous et al., 2004; Hajjar et al., 2006; Golebiowski et al., 2007). This protein was originally thought to be a Fisher strain variant denoted OBP1f (Briand et al., 2000). However, subsequent genome analysis has revealed a discrepancy in the original sequencing of OBP1 (Pevsner et al., 1990), indicating that the two proteins are identical. OBP1 is thought to be dimeric in solution at neutral $\mathrm{pH}$, having an apparent mass of $31.2 \mathrm{kDa}$ as determined by size-exclusion chromatography (Briand et al., 2000). However, OBP1 possesses a $\mathrm{C}$-terminal disulfide bond and thus in this regard is more akin to OBPp than to the domain-swapped OBPb. Here, we report the crystal structure of OBP1 to $1.6 \AA$ resolution and show that the protein is monomeric both in the crystalline form and in solution under native conditions. The structure of OBP1 represents the third mammalian OBP structure to be determined crystallographically.

\section{Materials and methods}

\subsection{Protein expression and purification}

Overexpression of OBP1 was performed in the methylotrophic yeast Pichia pastoris as described previously (Briand et al., 1999, 2000). Briefly, yeast cells containing the plasmid pA1fOBP were grown in buffered minimal glycerol media at $302 \mathrm{~K}$ and $250 \mathrm{rev} \mathrm{m^{-1 }}$ in baffled flasks for $2 \mathrm{~d}$. After this time, the $\mathrm{OD}_{600}$ had reached $\sim 50$ and the cells were gently harvested by centrifugation at $2000 \mathrm{rev} \mathrm{min}^{-1}$ for $5 \mathrm{~min}$. The cells were then resuspended in buffered minimal methanol media and incubated at $302 \mathrm{~K}$ and $250 \mathrm{rev} \mathrm{min}^{-1}$ in baffled flasks for $4 \mathrm{~d}$. Protein overexpression was driven by the metabolism of methanol, which was added twice daily.

After overexpression, the supernatant containing OBP1 was clarified by centrifugation at $10000 \mathrm{rev} \mathrm{min}^{-1}$ for $30 \mathrm{~min}$ at $277 \mathrm{~K}$ followed by sterile filtration $(0.22 \mu \mathrm{m}$, Sartorius Ministart High-Flow). Sodium azide was then added to the clarified supernatant to a final concentration of $0.2 \%(w / v)$ and the supernatant was incubated at room temperature for $30 \mathrm{~min}$. The supernatant was then dialysed $(12-14 \mathrm{kDa}$ MWCO Spectra/pore dialysis membrane) against $10 \mathrm{mM}$ tris(hydroxymethyl)aminomethane (Tris) buffer $\mathrm{pH} 8.0$ and $1 \mathrm{~m} M$ ethylenediaminetetraacetic acid (EDTA) for $3 \mathrm{~d}$. The dialysed supernatant was then loaded overnight onto a column (GE Healthcare XK 16/24) containing Q-Sepharose Fast Flow 
resin at a rate of approximately $1.0 \mathrm{ml} \mathrm{min} \mathrm{m}^{-1}$. Protein elution was achieved with a $0-0.5 \mathrm{M} \mathrm{NaCl}$ gradient at $1.0 \mathrm{ml} \mathrm{min} \mathrm{m}^{-1}$ over $100 \mathrm{ml}$. The location of OBP1 in the eluate was confirmed by SDS-PAGE. The pooled fractions containing OBP1 were then concentrated tenfold using a $5 \mathrm{kDa}$ MWCO Sartorius Vivacell and the concentrated protein was purified further on a column containing Superdex 75 (GE Healthcare) at a rate of $2.0 \mathrm{ml} \mathrm{min}{ }^{-1}$ buffered with $25 \mathrm{mM}$ ammonium acetate. OBP1 eluted as a single peak and protein integrity was determined by SDS-PAGE and mass spectrometry.

\subsection{Crystallization and data collection}

Gel-purified protein was dialysed (12-14 kDa MWCO Spectra/pore dialysis membrane) against copious volumes of $>16 \mathrm{M} \Omega$ Purite water before being concentrated using a Vivaspin 500, 5000 MWCO spin column (Sartorius). The concentrated protein was diluted to a final concentration of $10 \mathrm{mg} \mathrm{ml}^{-1}$ in $10 \mathrm{~m} M$ Tris buffer $\mathrm{pH} 7.5$. OBP1 crystals were grown at $291 \mathrm{~K}$ using the sitting-drop vapour-diffusion method, in which $200 \mathrm{nl}$ drops of a 1:1 mixture of protein and reservoir solutions were equilibrated against $100 \mu \mathrm{l}$ reservoir solution containing $100 \mathrm{~m} M$ imidazole $\mathrm{pH} 7.0,25 \%$ polyethylene glycol 5000, $100 \mathrm{mM} \mathrm{NaF}$ and 5\% ethylene glycol. For cryoprotection, crystals were briefly soaked in a series of reservoir solutions supplemented with an increasing concentration of ethylene glycol ( $5 \%$ steps) to a final concentration of $20 \%(v / v)$ before being flash-frozen at $100 \mathrm{~K}$. X-ray diffraction data (Table 1) were recorded on beamline ID14EH2 at the European Synchrotron Radiation Facility (ESRF) in Grenoble, France. The data were indexed, integrated and initially scaled using the program XDS (Kabsch, 1993); after conversion to the mtz format using the program POINTLESS (Evans, 2006), the data were further scaled and analysed with SCALA (Evans, 1997).

\subsection{Structure determination}

The structure was solved using molecular replacement (Phaser; McCoy et al., 2007) with an ensemble of all four subunits of the structure of the female hamster sex pheromone aphrodisin (PDB code 1e5p; Vincent et al., 2001) as a search model. The resulting phases together with the OBP1 aminoacid sequence were fed directly into the phase-improvement and autobuilding routines of RESOLVE (Terwilliger, 2004) to produce a bias-free starting model of 160 amino acids with backbones and side chains plus a further 73 amino acids with backbone only. The model was completed and refined using Coot (Emsley \& Cowtan, 2004) and phenix.refine (Afonine et al., 2005) to give a final model comprising residues 4-157 and 1-157 in subunits $A$ and $B$, respectively, 475 waters and five molecules of ethylene glycol. Overall, the electron-density map was of very high quality, except for amino acids 75-78 in chain $B$ owing to presumed mobility of the loop and lack of crystal contacts. In chain $A$, the corresponding loop is involved in several crystal contacts with a symmetry mate $(-x, y+1 / 2$, $-z+1 / 2)$. The cavities in each molecule had electron density for an extra ligand, but could not be interpreted.
Table 1

$\mathrm{X}$-ray data and refinement statistics.

Values in parentheses are for the highest resolution shell.

\begin{tabular}{|c|c|}
\hline \multicolumn{2}{|l|}{ Data-collection statistics $\dagger$} \\
\hline Unit-cell parameters $(\AA)$ & $\begin{array}{c}a=42.0, b=62.1, \\
c=109.2\end{array}$ \\
\hline Space group & $P 2_{1} 2_{1} 2_{1}$ \\
\hline Resolution $(\AA)$ & $42-1.6(1.69-1.60)$ \\
\hline Completeness (\%) & $98.4(93.0)$ \\
\hline Multiplicity & $3.4(2.6)$ \\
\hline$I / \sigma(I)$ & $17.4(4.7)$ \\
\hline No. of observations & 128811 (13633) \\
\hline No. of unique observations & $37976(5148)$ \\
\hline$R_{\text {merge }}$ & $4.6(22.0)$ \\
\hline \multicolumn{2}{|l|}{ Refinement statistics } \\
\hline \multicolumn{2}{|l|}{$B$ factors $\left(\AA^{2}\right)$} \\
\hline Average (all atoms) & 15.3 \\
\hline Wilson & 13.0 \\
\hline No. of non-H atoms & 2525 \\
\hline No. of waters & 475 \\
\hline R.m.s.d. bonds $(\AA)$ & 0.015 \\
\hline R.m.s.d. angles $\left({ }^{\circ}\right)$ & 1.288 \\
\hline \multicolumn{2}{|c|}{ Ramachandran plot, No. of non-Gly/Pro residues } \\
\hline Core & 258 \\
\hline Allowed & 24 \\
\hline Generously allowed & 1 \\
\hline Disallowed & 0 \\
\hline$R(\%)$ & 14.15 \\
\hline$R_{\text {free }} \ddagger(\%)$ & 18.10 \\
\hline PDB code & $3 \mathrm{fig}$ \\
\hline
\end{tabular}

$\dagger$ Statistics as reported by $S C A L A . \quad \ddagger 5 \%$ of reflections set aside for cross-validation.

\subsection{Analytical ultracentrifugation}

Sedimentation-velocity experiments were performed using a Beckman Coulter XL-A analytical ultracentrifuge. Samples contained $1.27 \mathrm{mg} \mathrm{ml}^{-1} \mathrm{OBP} 1$ in PBS with or without a twofold stoichiometric amount of linalool. Samples were loaded into two channel centrepieces and data were acquired at $280 \mathrm{~nm}$ in $5 \mathrm{~min}$ intervals at $40000 \mathrm{rev} \mathrm{min}^{-1}$ and $293 \mathrm{~K}$. Data were analysed using SEDFIT (Schuck et al., 2002) with the $c(M)$ and $c(S)$ models, assuming a frictional ratio of 1.2. The partial specific volume of OBP1 was calculated using SEDNTERP (Lebowitz et al., 2002). The density and viscosity of PBS were determined experimentally using an Anton Paar DMA 5000 densitometer. To determine the mass, the resulting $c(M)$ distribution tables were fitted to a single Gaussian distribution using SigmaPlot v. 8.0 (SPSS Inc., Chicago, Illinois USA).

\section{Results}

\subsection{Protein production, purification and crystallization}

OBP1 was overexpressed in the methylotrophic yeast $P$. pastoris as previously described (Briand et al., 2000). Mass spectrometry of the purified protein gave a molecular mass of $18131.06 \pm 0.21 \mathrm{Da}$ and was also used to gauge the purity of the starting material.

\subsection{The overall structure of OBP1}

The three-dimensional structure of OBP1 was solved by molecular replacement using aphrodisin (PDB code 1e5p; Vincent et al., 2001) as the starting structure. The structure of 
OBP1 has a fold typical of the lipocalin family, formed by eight antiparallel $\beta$-sheets with a contiguous topology and a C-terminal $\alpha$-helix that is packed against the exteriors of $\beta$-strands $\mathrm{A}, \mathrm{G}$ and $\mathrm{H}$ (Fig. 1). Each protein contains two disulfide bonds (Cys44-Cys48 and Cys64-Cys155) as assigned previously (Briand et al., 2000). Although two subunits are present in the crystallographic asymmetric unit, no evidence of dimerization was observed. Furthermore, the interfacial regions of monomers $A$ and $B$ have solvent-accessible areas of approximately 75 and $60 \AA^{2}$, respectively, of a total combined solvent-accessible area of approximately $15800 \AA^{2}$; this value of about $0.5 \%$ is far below that considered to be acceptable for solution dimers (Jones \& Thornton, 1995).

The N-terminal three residues of subunit $A$ are not visible in the electron density, probably owing to flexibility. Upon alignment of both monomers, the average root-mean-square deviation (r.m.s.d.) is $0.48 \AA$ between $147 \mathrm{C}^{\alpha}$ pairs, including Asn4-Lys58 and Gln64-Val150 (Fig. 2a). The r.m.s.d. is
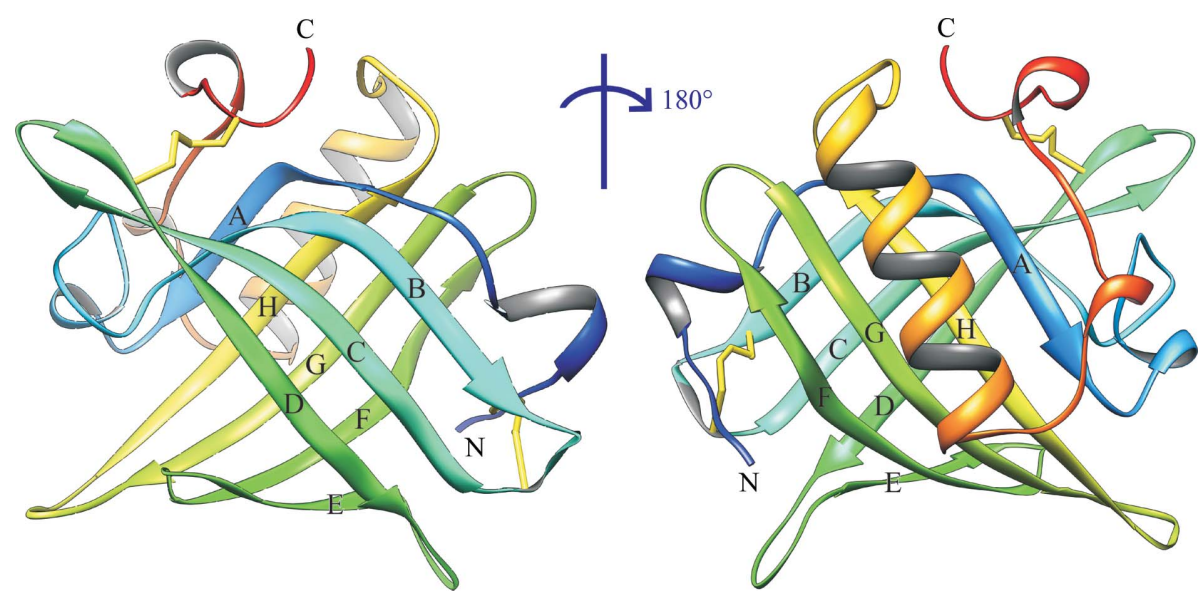

Figure 1

Ribbon diagram of the OBP1 monomer. The protein has been rotated through $180^{\circ}$ to view both sides. The structure is coloured from blue to red along the chain. The eight $\beta$-strands are labelled AH. Disulfide bonds are shown as sticks. This figure was created using UCSF Chimera (Pettersen et al., 2004).

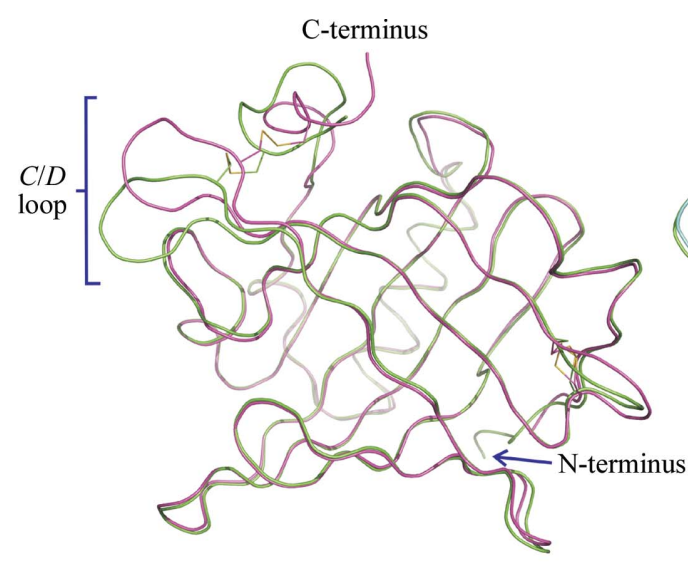

(a)

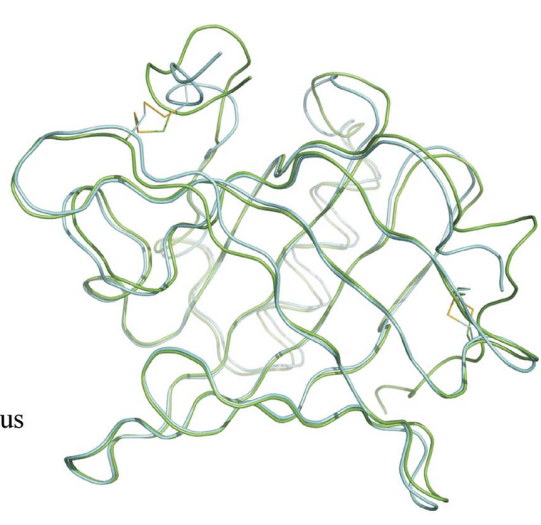

(b)
Figure 2

Alignments of OBP1 monomers $A$ and $B$ and of OBP1 with the OBP1 model. (a) An alignment of the two OBP1 monomers in the crystallographic asymmetric unit. (b) An alignment of OBP1 chain $B$ and a model of OBP1 made using the structure of aphrodisin (1e 5p chain $A$ ) as a template with SWISS-MODEL (Guex \& Peitsch, 1997). OBP1 monomers $A$ and $B$ and the modelled structure are coloured pink, green and blue, respectively. highest in the $C / D$ loop and the $\mathrm{C}$-terminus, probably owing to mobility. Since several different modelled structures of OBP1 have been reported in the literature (Nespoulous et al., 2004; Hajjar et al., 2006; Goleblowski et al., 2006), it was of interest to compare these with the crystal structure of OBP1 reported here. Accordingly, a model of the structure of OBP1 was prepared with the SWISS-MODEL server (Guex \& Peitsch, $1997)$ using the structure of aphrodisin (1e5p monomer $A$ ) as a template, as prepared by Nespoulous et al. (2004). As can be n the agreement is very high, with an r.m.s.d. of only $0.72 \AA$ 134 residue pairs of the OBP1 model and monomer $B$

\subsection{The internal binding cavity of OBP1}

Surface analysis of the structure of OBP1 using DeepView (Guex \& Peitsch, 1997) with a probe size of $1.4 \AA$ revealed the presence of a large internal cavity within each of the two monomers. This is in accordance with other lipocalins such as OBPp, aphrodisin and the mouse major urinary protein (MUP; Bocskei et al., 1992; Spinelli et al., 1998; Vincent et al., 2001). The computed cavity volumes for the $A$ and $B$ monomers of OBP1 are 326 and $328 \AA^{3}$, respectively, compared with 438 and $417 \AA^{3}$ for the $A$ and $B$ monomers of OBPp and volumes ranging from 238 to $345 \AA^{3}$ for the four monomers of aphrodisin that are found in the crystallographic asymmetric unit of this protein. Thus, the volume of the OBP1 calyx is approximately the same size of that of OBPp and of aphrodisin. However, it is prudent to mention that studies performed in silico have revealed that OBP cavity volumes can vary considerably (Hajjar et al., 2006; Goleblowski et al., 2006).

Electron density was found in the cavities of both of the OBP1 monomers (Fig. 3). However, after an exhaustive search loading monomers of every known component of the various reagents used during protein overexpression, purification and crystallization using the program Coot (Emsley \& Cowtan, 2004), nothing could be assigned to this density. This suggests that the binding pocket could be occupied by a pigment or a combination of different pigments that were produced by the yeast during protein overexpression. Native protein nanospray ionization mass spectrometry was used to try to determine the mass of the exogenous ligand, but no change in 

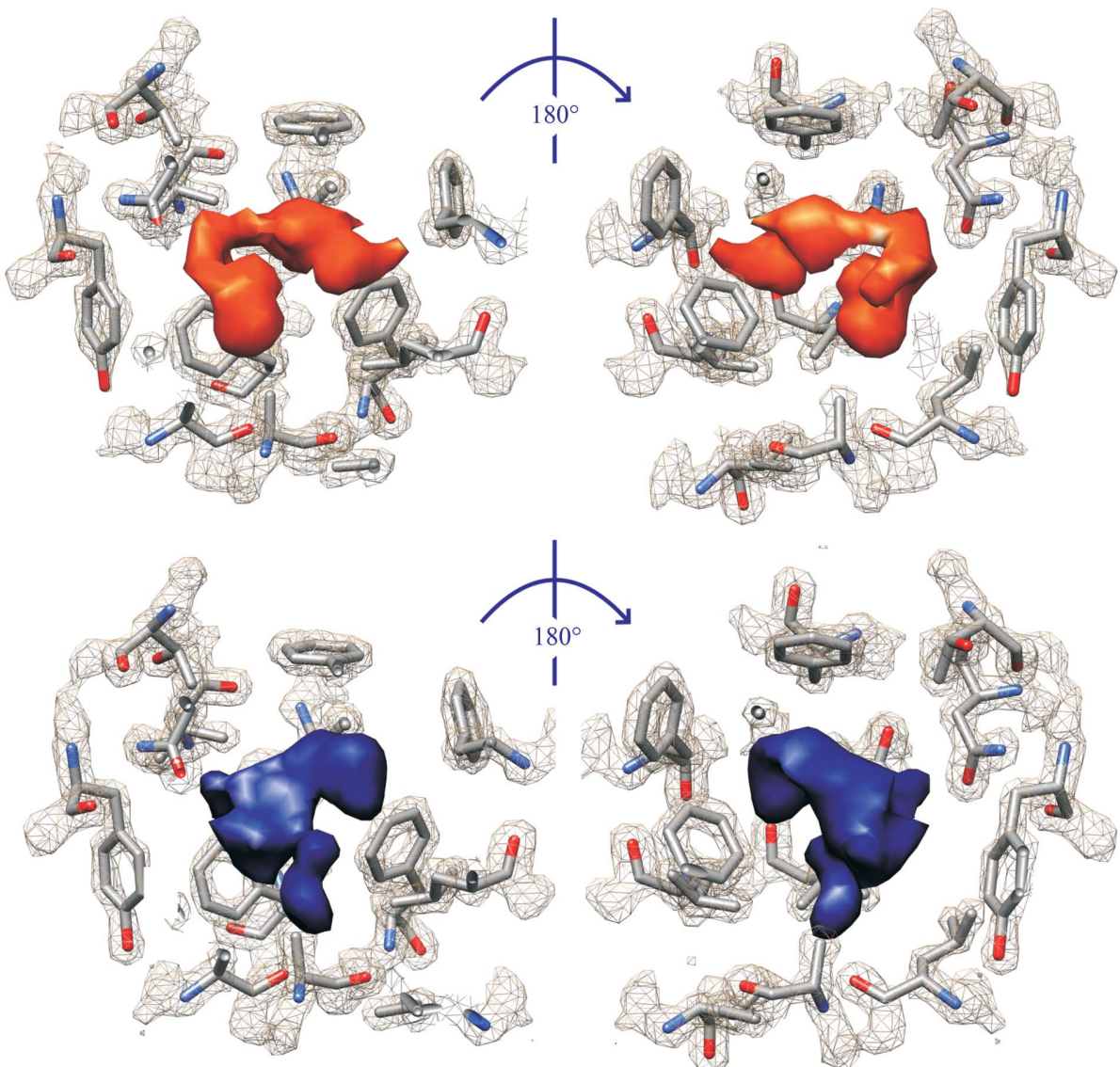

\section{1}
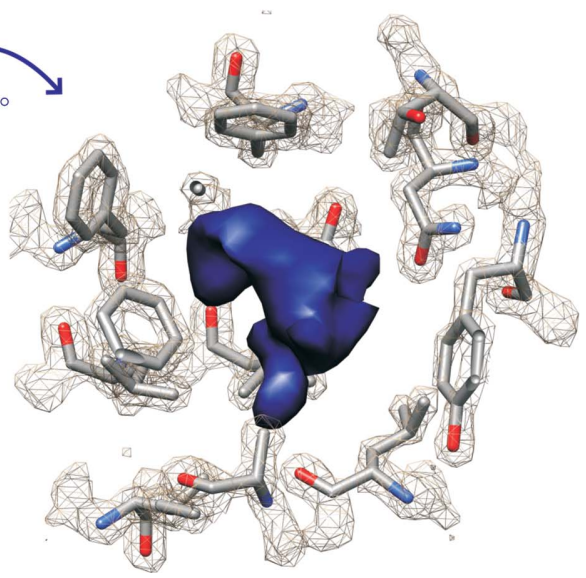

Figure 3

Unassigned density in the binding pockets of OBP1 monomer $A$ (orange) and OBP1 monomer $B$ (blue). Both the $F_{\mathrm{o}}-F_{\mathrm{c}}$ and the $2 F_{\mathrm{o}}-F_{\mathrm{c}}$ maps were normalized so that the r.m.s. noise was scaled to $1.0 \sigma$. The contour level of both maps is 2 and they were generated without surface smoothing using the program UCSF Chimera (Pettersen et al., 2004). Only the residues that contact the binding pocket are shown and those closest to the viewer have been clipped in order to better visualize the unassigned density. mass was observed for OBP1 in native and denaturing conditions (data not shown). This could suggest that the protein-ligand complex is unstable during ionization, a phenomenon that is commonly observed for hydrophobic interactions (Chung et al., 1998).

As in many other lipocalins (bovine $\beta$-lactoglobin being a notable exception), the internal cavity of OBP1 does not communicate directly with external solvent water. Thus, some form of rearrangement of the protein is thought to occur during ligand binding and unbinding. Experiments performed in silico with homology-modelled OBP1 have implied that Tyr82 plays a critical role in driving protein dynamics that permit access to the binding cavity (Hajjar et al., 2006; Goleblowski et al., 2006). Interestingly, upon analysis of the solvent-accessible surface area of the 15 residues that line the binding pocket of OBP1, Tyr82 was found to be significantly more solvent-exposed than any other residue. In accordance with this, direct solvent access to the binding cavity can most easily be achieved upon movement of Tyr82 (Fig. 4). As previously proposed, the conservation of an aromatic residue at position $82(\mathrm{Tyr}$ or Phe) in practically all lipocalins underlies the importance of this residue in bridging the external and internal

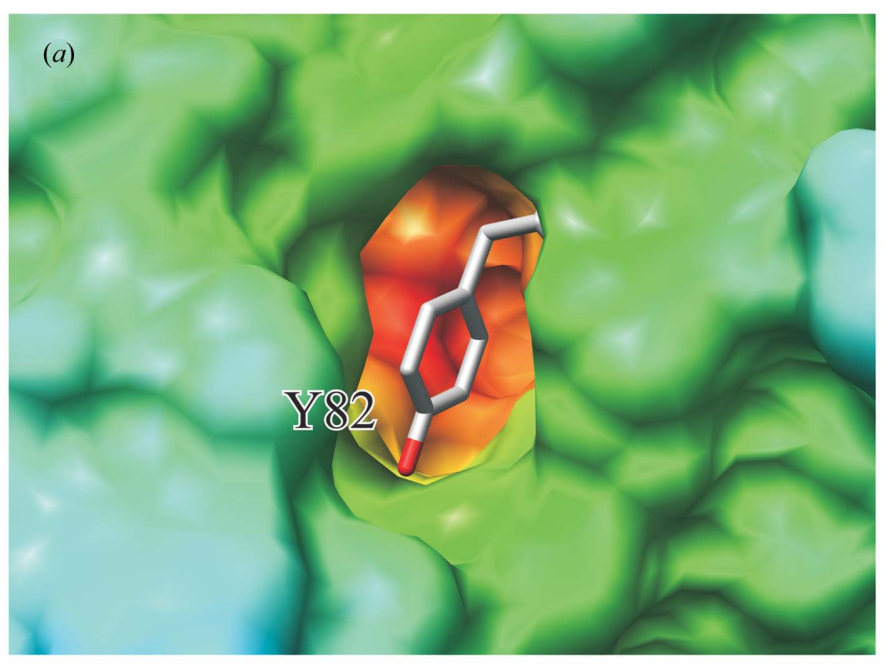

(b)

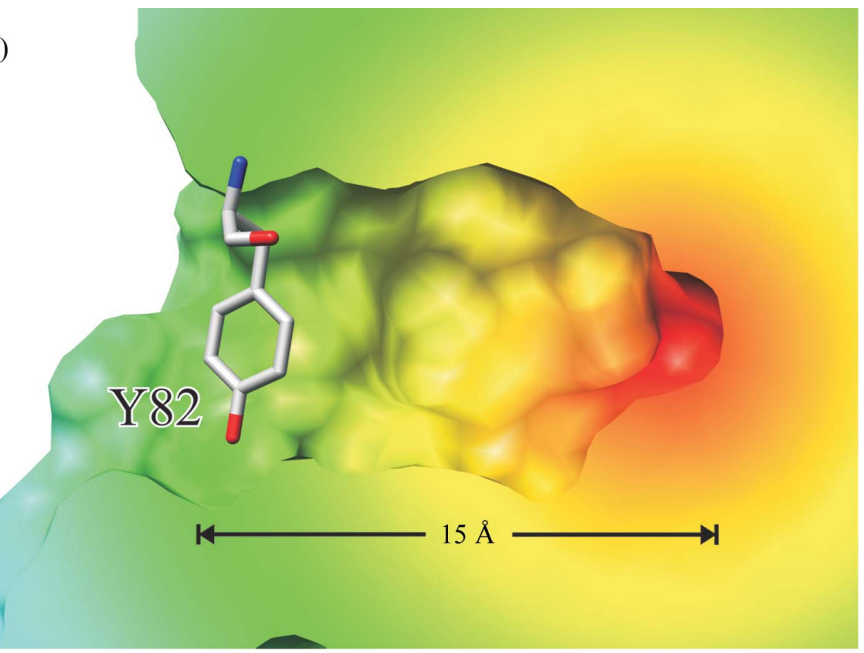

$\left.\right|_{0.0}$

Figure 4

Sliced view of OBP1 revealing the binding cavity. (a) The internal binding cavity of OBP1 is exposed on removal of Tyr82. (b) Rotation of $90^{\circ}$ around the $x$ axis reveals the depth of the OBP1 calyx. The protein surface is coloured as distance from the protein core. The scale bar is in $\AA$. 
environments. This residue could also govern suboptimal hydration of lipocalin-binding pockets, a process that has been implicated in imposing enthalpically driven ligand binding in MUP (Malham et al., 2005; Barratt et al., 2005).

\subsection{The oligomeric state of OBP1}

Previously, OBP1 had been assigned as being dimeric in solution based on size-exclusion chromatography, which gave an apparent weight of $31.2 \mathrm{kDa}$, a value almost twice that of the monomeric protein (Briand et al., 2000). This assignment was also supported by stoichiometries determined by isothermal titration calorimetry that were closer to equivalence when fitted to one binding site per protein dimer (Nespoulous et al., 2004). Velocity analytical ultracentrifugation (AUC) was performed in order to establish the oligomeric state of OBP1 (see §2). The $c(M)$ distributions of OBP1 with and without a twofold molar excess of linalool (provided in excess owing to the low binding stoichiometry reported for this ligand;

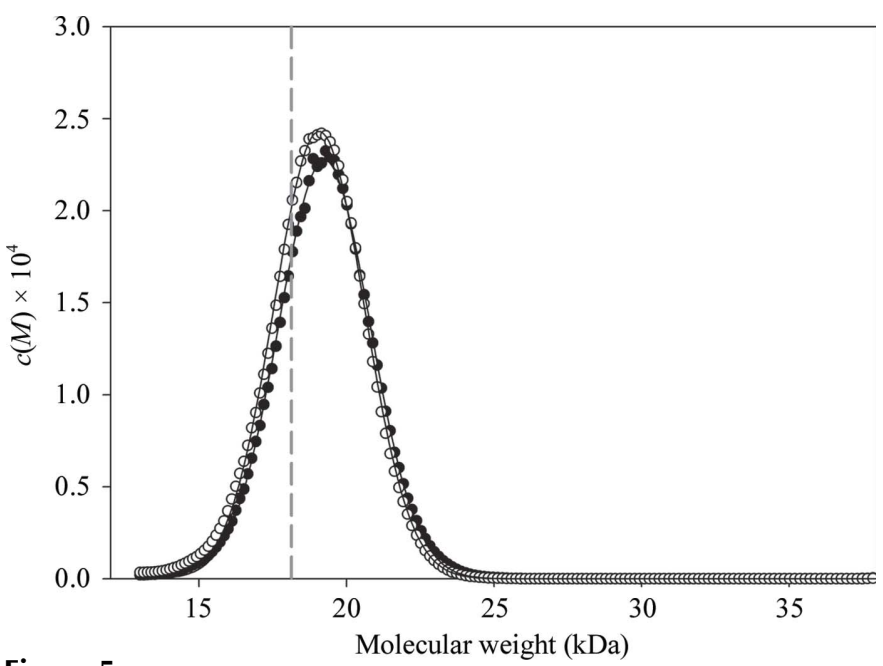

Figure 5

Sedimentation-velocity analytical ultracentrifugation of OBP1. $c(M)$ distributions of OBP1 with (closed circles) and without (open circles) the presence of a twofold molar excess of linalool are shown. The dashed line indicates the calculated mass of the apoprotein. Data were acquired using a Beckman-Coulter XL-A analytical ultracentrifuge. The protein concentration was $1.27 \mathrm{mg} \mathrm{ml}^{-1}$ in PBS.
Nespoulous et al., 2004) are monodisperse and have calculated molecular weights of 19068 and $19248 \mathrm{Da}$ for the samples in the absence and presence of linalool, respectively, values which are close to that of the monomeric protein (Fig. 5). In accordance with this, no evidence of protein dimerization was observed in the crystal structure of OBP1.

\subsection{Comparison of rat OBP subtypes}

The three different rat OBPs represent the only complete intraspecies OBP family for which comparisons have been made (Lobel et al., 2002). Interestingly, these proteins have been shown to possess different binding properties and specificities, providing the first evidence for OBP-dependent odour coding. It was thus considered of interest to make comparisons between the known binding-pocket residues of OBP1, reported here, with those of rat OBP2 and OBP3. Analysis of the crystal structure of OBP1 reveals that 15 different residues contact the binding pocket of this protein. Fig. 6 shows a sequence alignment of the three different rat OBP subtypes and demonstrates the apparent lack of homology in the binding pocket, providing insight into the origins of the different odorant specificities of these proteins.

\section{Discussion}

Interest in OBP1 is at least twofold. From a biological perspective the protein has significance because of its supposed role in olfaction, an area of research that has gained considerable momentum in a very short time (Firestein, 2001; Gilbert \& Firestein, 2002; Axel, 2005). It is hard to imagine that OBPs are not involved in olfaction in some way, given their physical location and the range of different volatile ligands that they bind. However, their place in olfactory processes is still based largely on assumptions gleaned primarily from thermodynamic binding information that has been gathered over the last ten years or so. Furthermore, we need to remind ourselves that the question of whether or not OBPs affect olfaction in some way is simply a precursor to the more interesting and important question of what they actually do. Thus, in this regard, there is still much work to be performed.

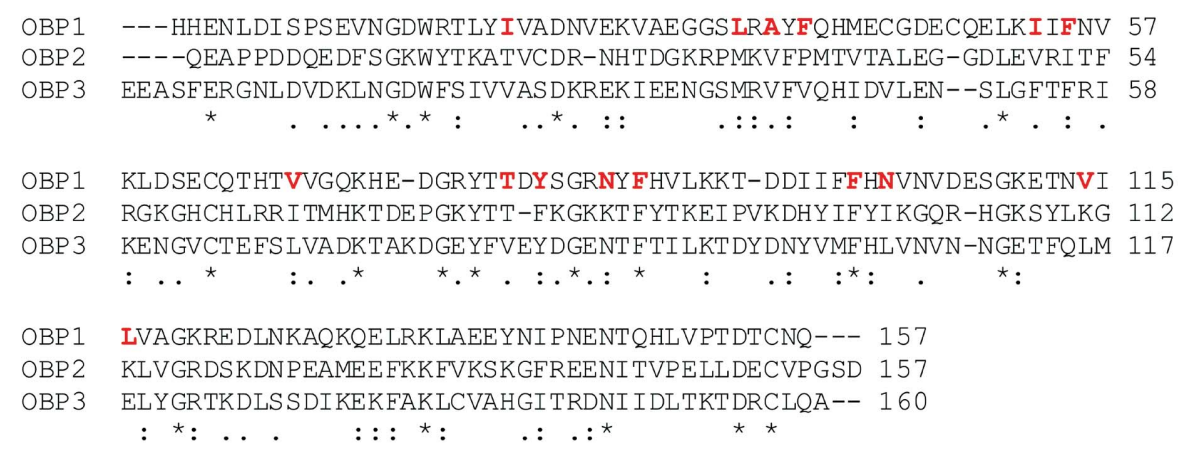

Figure 6

An alignment of the sequences of the three different rat OBP subtypes: OBP1, OBP2 and OBP3. Residues coloured red in the sequence of OBP1 are those that contact the binding pocket.
OBPs in general also present useful models to investigate fundamental problems such as hydrophobically driven ligand-protein interactions and protein dynamics. To this end, OBP1 is a useful conduit to help explore relatively new areas such as entropy-entropy compensation and binding-site hydration, both of which will have dramatic, albeit less apparent, consequences for novel drug-discovery programs (Bingham et al., 2004; Homans, 2007).

From the three-dimensional distribution of highly conserved residues, it is apparent that most are found at the 
opposite face of the protein to that of the $\mathrm{G} x \mathrm{~W}$ motif (Fig. 7). In fact, 11 of the 19 highly conserved residues (Glu27, Lys28, Val29, Glu31, Gly33, Arg36, Tyr78, Gly84, Asn86 and Phe88) are located in or around the $A / B$ and $E / F$ loops. In light of this, it may be instructive to note that the $A / B$ and the $E / F$ loops are widely regarded as being involved in the control of ligand binding and release (Golebiowski et al., 2007; Skerra, 2007). The alignment uncovers five invariant residues (Lys28, Tyr78, Gly84, Asn86 and Asn145); of these, Tyr78 has been shown to have a variable side chain $\mathrm{p} K_{\mathrm{a}}$ in the ligated and unligated protein (Nespoulous et al., 2004). At least two tyrosyl residues are thought to be involved in OR binding in vitro (Matarazzo et al., 2002). Asn86 is the only invariant residue that lines the binding pocket. In silico, Asn86 has been shown to act as a hydrogen-bond acceptor for ligands during unbinding simu-

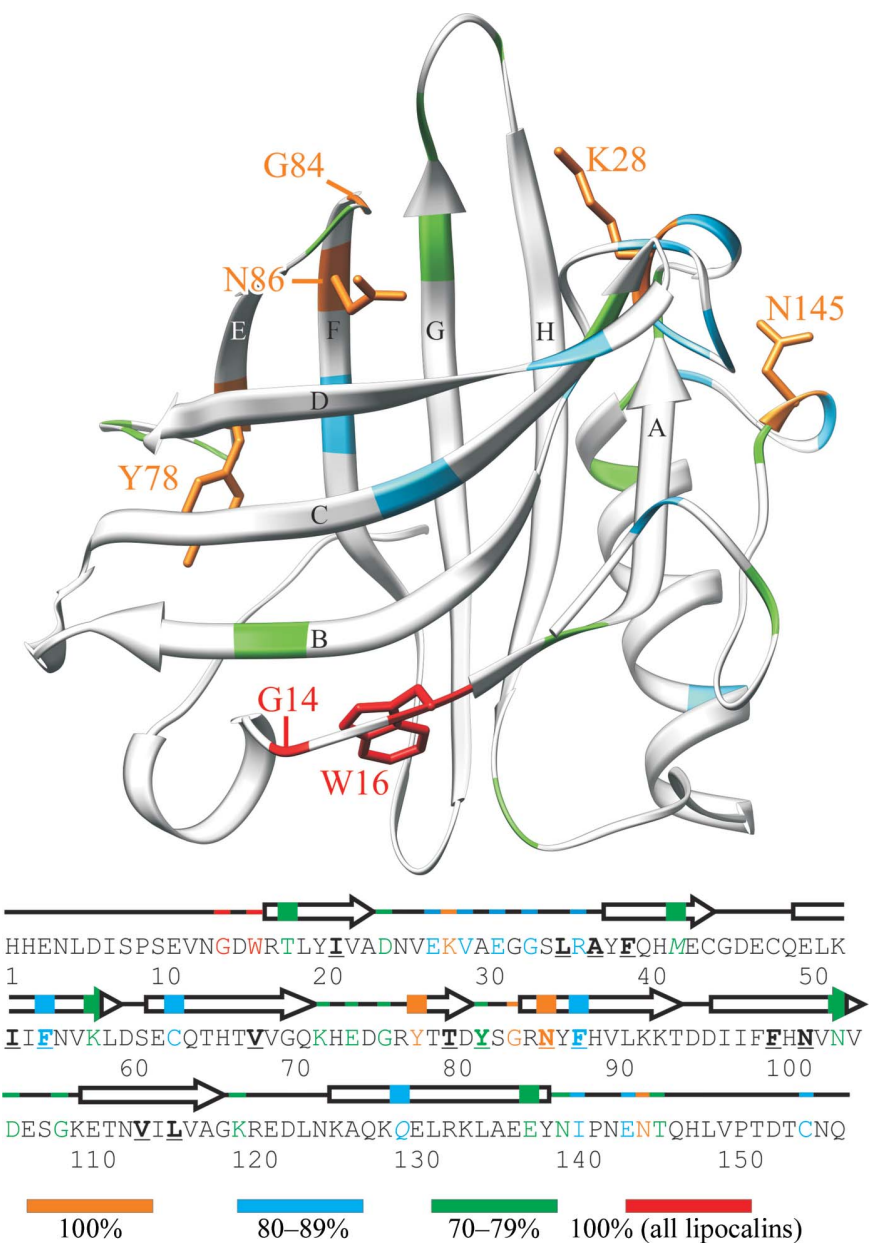

Figure 7

Sequence alignment of OBP1, aphrodisin, bovine lipocalin allergen (BLA), mouse major urinary protein (MUP), porcine OBP (OBPp), bovine $\mathrm{OBP}(\mathrm{OBPb})$ and major horse allergen (MHA) superimposed onto the structure of OBP1. In the ribbon diagram of OBP1 positions coloured orange, blue and green represent very highly conserved, highly conserved and moderately conserved residues, respectively (see key). Side chains of residues that are very highly conserved are shown, as are the residues of the $\mathrm{G} x \mathrm{~W}$ motif. Conserved residues are also coloured onto the sequence of OBP1 with units of secondary structure also shown. Residues shown in bold and underlined make contact with the binding cavity. Met42 and Gln130 are italicized; these residues are not conserved in OBP1. lations (Hajjar et al., 2006). The determination of the role of the five invariant residues in OBP1 should be a route towards significant enlightenment with respect to the structure-function relationship of this protein.

Tegoni and coworkers suggested that the shortened length of the loop preceding the $\alpha$-domain in $\mathrm{OBPb}$ places constraints on this domain that prevent it from packing against the parent monomer, thus driving dimerization (Spinelli et al., 1998; Ramoni et al., 2002). Akin to OBPp, OBP1 has a sixresidue loop preceding the $\alpha$-domain. It therefore seems that the length of this loop (as suggested by Tegoni and coworkers) may be critical in driving self-association. Furthermore, unlike $\mathrm{OBPb}$ but akin to OBPp, OBP1 possesses a C-terminal disulfide bond that may also hinder dimer formation by tethering the $\alpha$-domain to the parent barrel. Previous attempts to determine the oligomeric state of OBP1 using size-exclusion chromatography were proven to be incorrect. Whilst this has no bearing on olfactory processes since the biological role of this protein has not been established, the new monomeric assignment does have dramatic effects for the reported binding stoichiometries of OBP1. However, recent efforts in our laboratory to remove exogenous ligands after purification have resulted in binding stoichiometries close to one site per protein monomer (data not shown).

The crystal structure of OBP1 is consistent with those of all other monomeric lipocalins. Exhaustive interrogation of the high-resolution structure of OBP1 presented here will be extremely valuable and a good starting point for many future investigations.

We thank Jane Laughton and Dr Susan Liddell for help with the overexpression of OBP1 and mass spectrometry, respectively. We also thank Professor Jerome Golebiowski, Professor Arthur Rowe and Dr Thomas Gallagher for useful discussions. We are grateful to ESRF for travel and access to synchrotron facilities.

\section{References}

Afonine, P. V., Grosse-Kunstleve, R. W. \& Adams, P. D. (2005). CCP4 Newsl. 42, contribution 8.

Axel, R. (2005). Angew. Chem. Int. Ed. 44, 6110-6127.

Barratt, E., Bingham, R. J., Warner, D. J., Laughton, C. A., Phillips, S. E. \& Homans, S. W. (2005). J. Am. Chem. Soc. 127, 11827-11834.

Bianchet, M. A., Bains, G., Pelosi, P., Pevsner, J., Snyder, S. H., Monaco, H. L. \& Amzel, L. M. (1996). Nature Struct. Biol. 3, 934-939.

Bignetti, E., Cavaggioni, A., Pelosi, P., Persaud, K. C., Sorbi, R. T. \& Tirindelli, R. (1985). FEBS J. 149, 227-231.

Bingham, R. J., Findlay, J. B., Hsieh, S. Y., Kalverda, A. P., Kjellberg, A., Perazzolo, C., Phillips, S. E., Seshadri, K., Trinh, C. H., Turnbull, W. B., Bodenhausen, G. \& Homans, S. W. (2004). J. Am. Chem. Soc. 126, 1675-1681.

Bocskei, Z., Groom, C. R., Flower, D. R., Wright, C. E., Phillips, S. E., Cavaggioni, A., Findlay, J. B. \& North, A. C. (1992). Nature (London), 360, 186-188.

Briand, L., Eloit, C., Nespoulous, C., Bezirard, V., Huet, J. C., Henry, C., Blon, F., Trotier, D. \& Pernollet, J. C. (2002). Biochemistry, 41, 7241-7252. 
Briand, L., Nespoulous, C., Perez, V., Remy, J. J., Huet, J. C. \& Pernollet, J. C. (2000). FEBS J. 267, 3079-3089.

Briand, L., Perez, V., Huet, J. C., Danty, E., Masson, C. \& Pernollet, J. C. (1999). Protein Expr. Purif. 15, 362-369.

Chung, E., Henriques, D., Renzoni, D., Zvelebil, M., Bradshaw, J. M., Waksman, G., Robinson, C. V. \& Ladbury, J. E. (1998). Structure, 6, 1141-1151.

Cowan, S. W., Newcomer, M. E. \& Jones, T. A. (1990). Proteins, 8, 44-61.

Dal Monte, M., Andreini, I., Revoltella, R. \& Pelosi, P. (1991). Comp. Biochem. Physiol. B Biochem. Mol. Biol. 99, 445-451.

Emsley, P. \& Cowtan, K. (2004). Acta Cryst. D60, 2126-2132.

Evans, P. (2006). Acta Cryst. D62, 72-82.

Evans, P. R. (1997). Jnt CCP4/ESF-EACBM Newsl. Protein Crystallogr. 33, 22-24.

Firestein, S. (2001). Nature (London), 413, 211-218.

Firestein, S., Shepherd, G. M. \& Werblin, F. S. (1990). J. Physiol. 430, 135-158.

Flower, D. R., North, A. C. \& Sansom, C. E. (2000). Biochim. Biophys. Acta, 1482, 9-24.

Ganni, M., Garibotti, M., Scaloni, A., Pucci, P. \& Pelosi, P. (1997). Comp. Biochem. Physiol. B Biochem. Mol. Biol. 117, 287-291.

Gilbert, A. N. \& Firestein, S. (2002). Nature Neurosci. 5, Suppl., 10431045.

Goleblowski, J., Antonczak, S. \& Cabrol-Bass, D. (2006). J. Mol. Struct. Theochem, 763, 165-174.

Golebiowski, J., Antonczak, S., Fiorucci, S. \& Cabrol-Bass, D. (2007). Proteins, 67, 448-458.

Guex, N. \& Peitsch, M. C. (1997). Electrophoresis, 18, 2714-2723.

Hajjar, E., Perahia, D., Debat, H., Nespoulous, C. \& Robert, C. H. (2006). J. Biol. Chem. 281, 29929-29937.

Homans, S. W. (2007). Drug Discov. Today, 12, 534-539.

Jones, S. \& Thornton, J. M. (1995). Prog. Biophys. Mol. Biol. 63, 31-65.

Kabsch, W. (1993). J. Appl. Cryst. 26, 795-800.

Kontopidis, G., Holt, C. \& Sawyer, L. (2002). J. Mol. Biol. 318, 10431055.

Krautwurst D., Yau, K. W. \& Reed, R. R. (1998). Cell, 95, 917-926.

Lebowitz, J., Lewis, M. S. \& Schuck, P. (2002). Protein Sci. 11, 20672079.
Lobel, D., Jacob, M., Volkner, M. \& Breer, H. (2002). Chem. Senses, 27, 39-44.

Malham, R., Johnstone, S., Bingham, R. J., Barratt, E., Phillips, S. E., Laughton, C. A. \& Homans, S. W. (2005). J. Am. Chem. Soc. 127, 17061-17067.

Matarazzo, V., Zsurger, N., Guillemot, J. C., Clot-Faybesse, O., Botto, J. M., Dal Farra, C., Crowe, M., Demaille, J., Vincent, J. P., Mazella, J. \& Ronin, C. (2002). Chem. Senses, 27, 691-701.

McCoy, A. J., Grosse-Kunstleve, R. W., Adams, P. D., Winn, M. D., Storoni, L. C. \& Read, R. J. (2007). J. Appl. Cryst. 40, 658674.

Nespoulous, C., Briand, L., Delage, M. M., Tran, V. \& Pernollet, J. C. (2004). Chem. Senses, 29, 189-198.

Pettersen, E. F., Goddard, T. D., Huang, C. C., Couch, G. S., Greenblatt, D. M., Meng, E. C. \& Ferrin, T. E. (2004). J. Comput. Biol. 25, 1605-1612.

Pevsner, J., Hou, V., Snowman, A. M. \& Snyder, S. H. (1990). J. Biol. Chem. 265, 6118-6125.

Ramoni, R., Spinelli, S., Grolli, S., Conti, V., Merli, E., Cambillau, C. \& Tegoni, M. (2008). Biochim. Biophys. Acta, 1784, 651-657.

Ramoni, R., Vincent, F., Ashcroft, A. E., Accornero, P., Grolli, S., Valencia, C., Tegoni, M. \& Cambillau, C. (2002). Biochem. J. 365, 739-748.

Schuck, P., Perugini, M. A., Gonzales, N. R., Howlett, G. J. \& Schubert, D. (2002). Biophys. J. 82, 1096-1111.

Skerra, A. (2007). Curr. Opin. Biotechnol. 18, 295-304.

Spinelli, S., Ramoni, R., Grolli, S., Bonicel, J., Cambillau, C. \& Tegoni, M. (1998). Biochemistry, 37, 7913-7918.

Steinbrecht, R. A. (1998). Ann. NY Acad. Sci. 855, 323-332.

Taylor, A. J., Cook, D. J. \& Scott, D. J. (2008). Chem. Percept. 1, 153-162.

Tegoni, M., Ramoni, R., Bignetti, E., Spinelli, S. \& Cambillau, C. (1996). Nature Struct. Biol. 3, 863-867.

Terwilliger, T. (2004). J. Synchrotron Rad. 11, 49-52.

Vidic, J., Grosclaude, J., Monnerie, R., Persuy, M. A., Badonnel, K., Baly, C., Caillol, M., Briand, L., Salesse, R. \& Pajot-Augy, E. (2008). Lab Chip, 8, 678-688.

Vincent, F., Lobel, D., Brown, K., Spinelli, S., Grote, P., Breer, H., Cambillau, C. \& Tegoni, M. (2001). J. Mol. Biol. 305, 459469. 